\title{
An Energy-Efficient Scheme for Multirelay Cooperative Networks with Energy Harvesting
}

\author{
Dingcheng Yang, ${ }^{1}$ Chuanqi Zhu, ${ }^{1}$ Lin Xiao, ${ }^{1}$ Xiaomei Shen, ${ }^{2}$ and Tiankui Zhang ${ }^{2}$ \\ ${ }^{1}$ Information Engineering School, Nanchang University, Nanchang 330031, China \\ ${ }^{2}$ Beijing University of Posts and Telecommunications, Beijing, China \\ Correspondence should be addressed to Lin Xiao; xiaolin@ncu.edu.cn
}

Received 16 June 2016; Revised 23 October 2016; Accepted 20 November 2016

Academic Editor: Konstantinos Demestichas

Copyright (c) 2016 Dingcheng Yang et al. This is an open access article distributed under the Creative Commons Attribution License, which permits unrestricted use, distribution, and reproduction in any medium, provided the original work is properly cited.

\begin{abstract}
This study investigates an energy-efficient scheme in multirelay cooperative networks with energy harvesting where multiple sessions need to communicate with each other via the relay node. A two-step optimal method is proposed which maximizes the system energy efficiency, while taking into account the receiver circuit energy consumption. Firstly, the optimal power allocation for relay nodes is determined to maximize the system throughput; this is based on directional water-filling algorithm. Secondly, using quantum particle swarm optimization (QPSO), a joint relay node selection and session grouping optimization is proposed. With this algorithm, sessions can be classified into multiple groups that are assisted by the specific relay node with the maximum energy efficiency. This approach leads to a better global optimization in searching ability and efficiency. Simulation results show that the proposed scheme can improve the energy efficiency effectively compared with direct transmission and opportunistic relay-selected cooperative transmission.
\end{abstract}

\section{Introduction}

With the rapid growth of wireless communications, it is important to consider the costs in energy usage. This is especially true in cooperative networks where nodes are powered by batteries with finite capacities, as energy efficiency is critical because it affects the operation time of the network [1]. Hence, improving the energy efficiency for cooperative networks is essential to mitigate the limited energy available in nodes. Therefore, this gives us a good motivation to investigate the energy efficiency in cooperative networks.

Network-coded cooperation schemes are considered as a promising technology to improve energy efficiency and network throughput [2]. Network coding nodes combine multiple input data into one output stream to reduce the number of transmission time slots. However, the receiver circuit power consumption when network coding is used has not been rigorously considered before, an omission that is addressed in this paper.

Moreover, energy harvesting is a promising technique that can be used in relay nodes to increase flexibility and reduce the energy charge; it can bring more energy efficiency for wireless networks [3]. Most of the existing studies focus on power allocation schemes for relay nodes in a scenario with multiple unicast sessions. However, when using network coding, the network coding noise and receiver circuit power consumption increase as the size of session groups increases. Hence, there is a need to study how to perform relay node selection and session grouping for energy-efficient multirelay cooperative networks and that is the topic of this paper.

1.1. Related Work. Research about network-coded cooperative networks to improve network throughput is considered in [4-8]. In [3], the concept of network coding noise which hinders the performance of the overall network was first introduced. In [5], the authors analyzed the origin of the noise by studying signal aggregation at relay nodes and signal extraction at destination nodes. In [6], two novel closed-form power allocation scheme was proposed to reduce network coding noise in two-unicast wireless systems. Based on [6], a joint group assignment and power allocation algorithm were studied in [7]; this aims to reduce network coding noise in a multiunicast cooperative network. Furthermore, the relay 
selection and session grouping problem was investigated in [8], as session grouping can effectively reduce network coding noise. However, in all of these works, the circuit energy consumption with network coding was not considered.

To achieve better tradeoff between network throughput and power consumption, the metric energy efficiency is used in [9-11]. In [9], the authors studied the energy-efficient relay selection and power allocation problem for a twoway relay channel based on network coding. Paper [10] presented an opportunistic listening scheme to promote energy efficiency for network-coded cooperative networks in two-unicast sessions. Paper [11] proposed an energy-efficient multicast scheme with network coding.

Moreover, energy harvesting technologies for cooperative networks have been investigated in [12-17] and these represent a promising approach for powering nodes. In [12], an opportunistic wireless information and energy transfer relaying scheme was proposed by Lagrangian optimality. Reference [13] used the directional water-filling algorithm to maximize the sum-rate.

In cooperative relay networks with energy harvesting, the relay nodes selected and the choice of power allocation scheme have a great impact on the system performance. In [14], finite energy storage was considered in relay nodes and a relay selection scheme was proposed based on finite-state Markov chain. In $[15,16]$, the source nodes and relay nodes were considered as energy harvesting nodes, and then the system throughput was maximized by optimizing both relay selection and power allocation.

Another line of work on joint wireless energy and information transmission in relay networks is simultaneous wireless information and power transfer (SWIPT). Researchers have focused on improving energy efficiency and a particularly relevant piece of work is [17]. To maximize the total energy efficiency, the authors proposed a joint relay selection and resource allocation scheme for SWIPT relay networks with multiple source nodes and destination nodes pairs and energy harvesting relay nodes.

Improving the energy efficiency in relay networks is of critical importance for resolving the energy shortage of nodes. In the literature [14-17], the energy arrival time and the amount of harvested energy are known prior to transmission. However, in real systems, the amount of the harvested energy is affected by many factors, such as weather and channel conditions, so it is necessary to consider a transmission scheme that can efficiently use the harvested energy from random energy sources.

1.2. Contributions and Organization. In this paper, we focus on a multirelay cooperative network, where relay nodes are powered by random energy sources. Unlike previous works, our study considers network coding, finite energy storage, and energy harvesting. The aim of this paper is to optimize energy efficiency in multirelay cooperative networks with energy harvesting. In [18], we have proposed an energyefficient session grouping scheme in a single relay networkcoded cooperative network. In this work, we extend our previous work to a multirelay cooperative network and consider the energy harvesting technology.

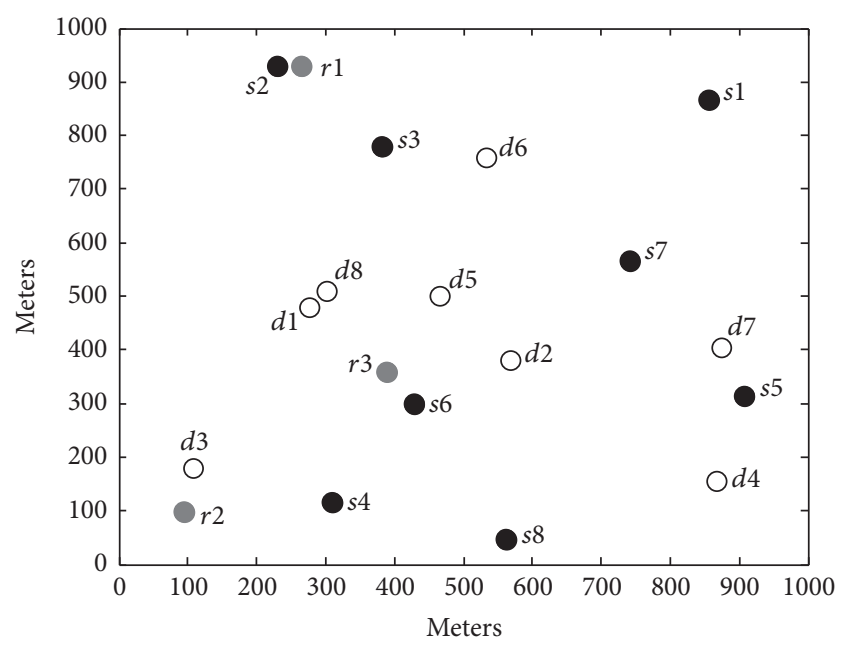

FIGURE 1: Multirelay cooperative network model.

Our contributions are concluded in three aspects:

(i) The receiver circuit power consumption with network coding is analyzed.

(ii) An optimal power allocation for relay nodes is proposed based on the directional water-filling algorithm.

(iii) An energy-efficient relay selection and session grouping scheme is proposed based on the quantum particle swarm optimization (QPSO).

The rest of this paper is organized as follows. Section 2 presents the network model including the throughput model and power consuming model. Section 3 studies the optimal power allocation scheme and jointly optimizing multirelay selection and session grouping scheme. Section 4 shows the numerical results and analysis for the proposed scheme. Section 5 is the conclusion of this paper.

\section{Network Model}

As is depicted in Figure 1, we consider a network-coded cooperative network which consists of $N$ sessions and $K$ relay nodes in a square with dimensions $D \times D$. In this model, we assume that the relay nodes operate in amplify-and-forward (AF) mode and use orthogonal channels. Sessions that select the same node share one channel and transmit data under time division multiple access (TDMA). Moreover, the relay nodes can harvest energy from random energy sources, such as wind energy and solar energy.

2.1. Total Throughput. In cooperative relaying networks, there are three transmission schemes: (i) direct transmission, (ii) opportunistic relay-selected cooperative transmission, and (iii) network-coded cooperative transmission. The transmitting power of relay nodes is not constant since the random energy arrivals in the relay nodes.

In network-coded cooperative networks, sessions in a group are assumed to be relayed in equal power. $P_{r_{k}}^{m}$ is defined 
as the transmit power of relay node $r_{k}$ for group $m$. The amplification factor $\alpha_{r_{k}}^{m}$ for $r_{k}$ is expressed as

$$
\left|\alpha_{r_{k}}^{m}\right|^{2}=\frac{P_{r_{k}}^{m}}{\left|G_{m}^{r_{k}}\right| \sigma_{r_{k}}^{2}+\sum_{s_{l} \in G_{m}^{r_{k}}} P_{s_{l}}\left|h_{s_{l} r_{k}}\right|^{2}},
$$

where $G_{m}^{r_{k}}$ denotes the set of sessions in group $m$ for $r_{k},\left|G_{m}^{r_{k}}\right|$ is the cardinality of $G_{m}^{r_{k}}$, and $P_{s_{l}}$ captures the transmit power of source node $s_{l}$.

Under network-coded cooperative networks, there exits network coding noise (NC noise) at each destination node, when extracting desired data from the combined data. We assume that a session $\left(s_{n}, d_{n}\right)$ is in a group $G_{m}^{r_{k}}$, so the NC noise $z_{d_{n}}^{\mathrm{NC}}$ at $d_{n}$ is expressed as

$$
z_{d_{n}}^{\mathrm{NC}}=z_{d_{n}}+\sum_{s_{l} \in G_{m}^{r_{k}}}^{s_{l} \neq s_{n}} \alpha_{r_{k}} h_{r_{k} d_{n}} z_{r_{k}}-\sum_{s_{l} \in G_{m}^{r_{k}}}^{s_{l} \neq s_{n}} \frac{\alpha_{r_{k}} h_{r_{k} d_{n}} h_{s_{l} r_{k}}}{h_{s_{l} d_{n}}} z_{d_{n}}
$$

where $z_{d_{n}}$ and $z_{r_{k}}$ are the Gaussian noise at $d_{n}$ and $r_{k}$, respectively, and $h_{u v}$ denotes the channel gain between node $u$ and node $v$.

Suppose that the channel between all nodes follows a simplified channel model that combines the distancedependent signal attenuation and long term shadowing with loss exponent $\alpha>2$. Thus, $h_{u v}$ can be expressed as $h_{u v}=$ $\|u-v\|^{-\alpha}$, where $\|u-v\|$ represents the distance between node $u$ and node $v$ and $\alpha$ is the channel loss exponent. From (2), the variance of the $\mathrm{NC}$ noise at $d_{n}$ is shown as

$$
\begin{aligned}
\sigma_{d_{n}^{\mathrm{NC}}}^{2}= & \sigma_{d_{n}}^{2}+\left(\left|G_{m}^{r_{k}}\right|-1\right)\left(\alpha_{r_{k}}^{m} h_{r_{k} d_{n}}\right)^{2} \sigma_{r_{k}}^{2} \\
& +\sigma_{d_{n}}^{2} \sum_{s_{l} \in G_{m}^{r_{k}}}^{s_{l} \neq s_{n}}\left(\frac{\alpha_{r_{k}}^{m} h_{s_{l} r_{k}} h_{r_{k} d_{n}}}{h_{s_{l} d_{n}}}\right)^{2} .
\end{aligned}
$$

A session has the option to select a relay node from different available relay nodes in our network. In addition, each session may use at most one relay node. We assume that $N$ sessions are sorted into $M$ groups, each session belongs to only one group. For notational simplicity, $G_{0}^{r_{k}}$ is defined as the direct transmission group by using the channel of $r_{k}$. We consider data transmission for each group ( $\left|G_{m}^{r_{k}}\right|$ sessions) in a time frame $T$, which is equally divided into $\left|G_{m}^{r_{k}}\right|+1$ time slots. For the first $\left|G_{m}^{r_{k}}\right|$ time slots, source nodes transmit data one by one, while the relay node and each destination node keep receiving data from all source nodes. At the last time slot, $r_{k}$ transmits the combined data, which is received by each destination node to extract desired data. $t_{n}$ is defined as the time duration of each session; then, we have

$$
t_{n}=\frac{T}{\sum_{m=0}^{M}\left|G_{m}^{r_{k}}\right|} \cdot \frac{\left|G_{m}^{r_{k}}\right|}{\left|G_{m}^{r_{k}}\right|+1}
$$

Thus, the throughput for a session $\left(s_{n}, d_{n}\right)$ is shown as

$$
\begin{aligned}
R_{\mathrm{NC}}\left(s_{n}, r_{k}, G_{m}^{r_{k}}\right)= & \frac{t_{n} \cdot W \cdot I_{\mathrm{NC}}\left(s_{n}, r_{k}, G_{m}^{r_{k}}\right)}{T} \\
= & \frac{W}{\sum_{m=0}^{M}\left|G_{m}^{r_{k}}\right|} \times \frac{\left|G_{m}^{r_{k}}\right|}{\left|G_{m}^{r_{k}}\right|+1} \\
& \times I_{\mathrm{NC}}\left(s_{n}, r_{k}, G_{m}^{r_{k}}\right),
\end{aligned}
$$

where $\left(s_{n}, d_{n}\right) \in G_{m}^{r_{k}}$ and $W$ is the bandwidth of each relay node. $I_{\mathrm{NC}}\left(s_{n}, r_{k}, G_{m}^{r_{k}}\right)$ is the mutual information for a session $\left(s_{n}, d_{n}\right)$; it is expressed as

$$
\begin{aligned}
I_{\mathrm{NC}}\left(s_{n}, r_{k}, G_{m}^{r_{k}}\right)=\log _{2}\left(1+\gamma_{s_{n} d_{n}}\right. \\
\left.+\frac{\gamma_{s_{n} r_{k}} \gamma_{r_{k} d_{n}}}{\left|G_{m}^{r_{k}}\right|\left(\sigma_{d_{n}^{\mathrm{NC}}}^{2} / \sigma_{d_{n}}^{2}\right)+\gamma_{r_{k} d_{n}}+\left(\sigma_{d_{n}^{\mathrm{NC}}}^{2} / \sigma_{d_{n}}^{2}\right) \sum_{s_{l} \in G_{m}^{r_{k}} \gamma_{s_{l} r_{k}}}}\right),
\end{aligned}
$$

where $\gamma_{s_{n} d_{n}}=\left(P_{s_{n}} / \sigma_{d_{n}}^{2}\right)\left|h_{s_{n} d_{n}}\right|^{2}, \gamma_{s_{n} r_{k}}=\left(P_{s_{n}} / \sigma_{r_{k}}^{2}\right)\left|h_{s_{n} r_{k}}\right|^{2}$, and $\gamma_{r_{k} d_{n}}=\left(P_{r_{k}} / \sigma_{d_{n}}^{2}\right)\left|h_{r_{k} d_{n}}\right|^{2}$. From (5) and (6), the throughput for a session $\left(s_{n}, d_{n}\right)$ under network-coded cooperative transmission scheme is

$$
\begin{aligned}
& R_{\mathrm{NC}}\left(s_{n}, r_{k}, d_{n}\right)=\frac{W}{\sum_{m=0}^{M}\left|G_{m}^{r_{k}}\right|} \times \frac{\left|G_{m}^{r_{k}}\right|}{\left|G_{m}^{r_{k}}\right|+1} \times \log _{2}(1 \\
& +\gamma_{s_{n} d_{n}} \\
& \left.+\frac{\gamma_{s_{n} r_{k}} \gamma_{r_{k} d_{n}}}{\left|G_{m}^{r_{k}}\right|\left(\sigma_{d_{n}^{\mathrm{NC}}}^{2} / \sigma_{d_{n}}^{2}\right)+\gamma_{r_{k} d_{n}}+\left(\sigma_{d_{n}^{\mathrm{NC}}}^{2} / \sigma_{d_{n}}^{2}\right) \sum_{s_{l} \in G_{m}^{r_{k}} \gamma_{s_{l} r_{k}}}}\right) .
\end{aligned}
$$

Similar to our previous work, for opportunistic cooperative communication scheme and network-coded cooperative communication without grouping scheme, throughput (7) is also appropriate when $\left|G_{m}^{r_{k}}\right|=1$ and $\left|G_{m}^{r_{k}}\right|=N$, respectively [18]. Under direct transmission scheme, the throughput for $\left(s_{n}, d_{n}\right)$ is

$$
R_{D}\left(s_{n}, r_{k}, d_{n}\right)=\frac{W}{\sum_{m=0}^{M}\left|G_{m}^{r_{k}}\right|} \times \log \left(1+s_{n} d_{n}\right) .
$$

2.2. Power Consumption. In this part, we derive the expression of total power consumption in network-coded cooperative networks.

We denote the transmission power for source nodes as $P_{s}$. Assuming that the transmitting circuit power $P_{c t}$ and the receiver circuit power $P_{\mathrm{cr}}$ are the same, for a group in network-coded cooperative networks with grouping scheme, the destination nodes keep receiving data, while the relay node keeps receiving data except for the last slot. The receiving time for each destination node in a group $G_{m}^{r_{k}}$ is

$$
t_{d-\mathrm{cr}}=\frac{T}{\sum_{m=0}^{M}\left|G_{m}^{r_{k}}\right|} \cdot\left|G_{m}^{r_{k}}\right|
$$


Thus, in a time frame $T$, the total receiver circuit energy consumption at destination nodes is

$$
E_{d_{-} \mathrm{cr}}\left(G_{m}^{r_{k}}\right)=t_{d_{-} \mathrm{cr}} P_{\mathrm{cr}}\left|G_{m}^{r_{k}}\right| .
$$

Let $t_{r \text {-cr }}$ denote the receiving time of relay node $r_{k}$ for $G_{m}^{r_{k}}$; then, we have

$$
t_{r-\mathrm{cr}}=\frac{T}{\sum_{m=0}^{M}\left|G_{m}^{r_{k}}\right|} \cdot \frac{\left|G_{m}^{r_{k}}\right|^{2}}{\left|G_{m}^{r_{k}}\right|+1} .
$$

We assume that the transmission time $t_{\text {send }}$ of source nodes and relay nodes for $G_{m}^{r_{k}}$ are the same; it is shown as

$$
t_{\text {send }}=\frac{T}{\sum_{m=0}^{M}\left|G_{m}^{r_{k}}\right|} \cdot \frac{\left|G_{m}^{r_{k}}\right|}{\left|G_{m}^{r_{k}}\right|+1} .
$$

Thus, the total energy consumption for the source nodes and relay nodes can be written as follows, respectively:

$$
\begin{aligned}
E_{s_{t}}\left(G_{m}^{r_{k}}\right) & =\left(P_{s}+P_{\mathrm{ct}}\right) \cdot t_{\text {send }} \cdot\left|G_{m}^{r_{k}}\right|, \\
E_{r_{k \text { sum }}}\left(G_{m}^{r_{k}}\right) & =\left(P_{r_{k}}^{m}+P_{\mathrm{ct}}\right) \cdot t_{\text {send }}+P_{\mathrm{cr}} \cdot t_{r_{\mathrm{cr}}} .
\end{aligned}
$$

Until now, the total power consumption for transmitting $G_{m}^{r_{k}}$ is

$$
P\left(G_{m}^{r_{k}}\right)=\frac{E_{d_{-} \mathrm{cr}}\left(G_{m}^{r_{k}}\right)+E_{s\lrcorner}\left(G_{m}^{r_{k}}\right)+E_{r_{k-s u m}}\left(G_{m}^{r_{k}}\right)}{T},
$$

$$
m \geq 1 \text {. }
$$

From (14), the total power consumption consists of three main components: source nodes, relay nodes, and destination nodes. From (11)-(13), $P\left(G_{m}^{r_{k}}\right)$ can be rewritten as follows:

$$
\begin{aligned}
& P\left(G_{m}^{r_{k}}\right)=\frac{1}{\sum_{m=0}^{M}\left|G_{m}^{r_{k}}\right|} \cdot \frac{\left|G_{m}^{r_{k}}\right|^{2}}{\left|G_{m}^{r_{k}}\right|+1} \\
& \quad\left(P_{s}+\left(1+\frac{1}{\left|G_{m}^{r_{k}}\right|}\right) P_{\mathrm{ct}}+\left(2+\left|G_{m}^{r_{k}}\right|\right) P_{\mathrm{cr}}+\frac{P_{r_{k}}^{m}}{\left|G_{m}^{r_{k}}\right|}\right) .
\end{aligned}
$$

For opportunistic cooperative communication scheme and network-coded cooperative communication without grouping scheme, the total receiver circuit power consumption for a group $G_{m}^{r_{k}}$ is obtained by substituting $\left|G_{m}^{r_{k}}\right|=1$ and $\left|G_{m}^{r_{k}}\right|=N$ into (15). For the direct transmission group, each destination node keeps receiving data for its time slot. Hence, the power consumption of direct transmission group can be expressed as

$$
P\left(G_{0}^{r_{k}}\right)=\frac{\left|G_{0}^{r_{k}}\right|}{\sum_{m=0}^{M}\left|G_{m}^{r_{k}}\right|} \cdot\left(P_{\mathrm{cr}}+P_{s}+P_{\mathrm{ct}}\right) .
$$

Combining (15) and (16), the expression of total power consumption in network-coded cooperative networks is

$$
P_{\text {total }}=\sum_{k=1}^{K} \sum_{m=0}^{M-1} P\left(G_{m}^{r_{k}}\right) \text {. }
$$

\section{Joint Optimization of Relay Selection and Session Grouping}

In this section, we divide the energy efficiency problem into two subproblems: the power allocation problem and the energy-efficient transmission optimization problem. For the first problem, an energy allocation scheme is proposed based on directional water-filling structure. For the second problem, we use quantum particle swarm optimization (QPSO) and propose a new algorithm which jointly considers relay selection problem and session grouping based on the solution of the first problem.

3.1. Optimal Power Allocation. The conventional water-filling algorithm is a useful technique in wireless communication for allocating power between different channels. It implies allocating more power to the channel with the higher SNR. In [19], a directional water-filling algorithm was introduced which considers the causality constraints on the energy usage. In this paper, we solve the optimal power allocation problem based on directional water-filling.

The relay nodes harvest energy from unpredictable energy sources, such as solar energy and wind energy which greatly depend on the conditions of the environment.

We assume energy arrivals $E_{1}, E_{2}, \ldots, E_{N}$ occur in the time instants $t_{1}, t_{2}, \ldots, t_{N}$ by a deadline $T$; the duration of each slot is $L_{n}=t_{n}-t_{n-1}$. Let $t_{0}=0$ for simplicity. The transmission rates are affected by the incoming energy. The objective is to maximize the throughput by optimizing power allocation for relay nodes.

It is assumed that the transmit power of each relay is constant within each slot. Each relay node is equipped with an energy receiver. The harvested energy is stored in a rechargeable battery. Thus, the energy causality constraints are obtained as follows:

$$
\begin{gathered}
\sum_{n=1}^{n} L_{n} P_{n} \leq \sum_{n=0}^{n-1} E_{i}, \quad n=1,2, \ldots, N+1, \\
\sum_{n=0}^{n} E_{n}-\sum_{n=1}^{n} L_{n} P_{n} \leq E_{\max }, \quad n=1, \ldots, N,
\end{gathered}
$$

where $P_{n}$ denotes the transmit power of relay nodes during slot $L_{n}$ and $E_{\max }$ is the storage capacity of the battery at relay nodes. It is also assumed that $E_{0}$ is the initial battery energy and $E_{0}>0$. Then, the optimization problem can be formulated as

$$
\begin{array}{ll}
\max _{P_{n} \geq 0} & \sum_{n=1}^{N+1} \frac{W L_{n}}{2} \log \left(1+P_{n}\right) \\
\text { s.t. } & \sum_{n=1}^{n} L_{n} P_{n} \leq \sum_{n=0}^{n-1} E_{n}, \quad n=1,2, \ldots, N+1 \\
& \sum_{n=0}^{n} E_{n}-\sum_{n=1}^{n} L_{n} P_{n} \leq E_{\max }, \quad n=1, \ldots, N .
\end{array}
$$

Since the objective function in (20) is not convex, constraint (18) and constraint (19) are linear in $P_{n}$, which are 
convex functions. Hence, the above optimization problem is a convex optimization problem. We define the Lagrangian function as follows:

$$
\begin{aligned}
\varsigma= & \sum_{n=1}^{N+1} \frac{W L_{n}}{2} \log \left(1+P_{n}\right) \\
& -\sum_{n=1}^{N+1} \tau_{n}\left(\sum_{n=1}^{n} L_{n} P_{n}-\sum_{n=0}^{n-1} E_{n}\right),
\end{aligned}
$$

where $\tau_{n}$ is the Lagrangian multipliers and $\tau_{n}>0$. Additional complimentary slackness conditions can be expressed as follows:

$$
\begin{aligned}
\tau_{n}\left(\sum_{n=1}^{n} L_{n} P_{n}-\sum_{n=0}^{n-1} E_{n}\right) & =0, \quad n=1,2, \ldots, N \\
\eta_{n}\left(\sum_{n=0}^{n} E_{n}-\sum_{n=1}^{n} L_{n} P_{n}-E_{\max }\right) & =0, \quad n=1,2, \ldots, N .
\end{aligned}
$$

Since the constraint is satisfied with equality, increasing $P_{n}$ will increase the objective function. Then, the optimal transmit power during each slot can be obtained by applying the KKT optimality conditions:

$$
P_{n}^{*}=\frac{1}{\sum_{j=n}^{N+1} \tau_{n}-\sum_{j=n}^{N} \eta_{n}}-1, \quad n=1, \ldots, N
$$

and $P_{N+1}^{*}=1 / \tau_{N+1}-1 . P_{n}^{*}$ is unique which satisfies $\sum_{n=1}^{n} L_{n} P_{n}=\sum_{n=0}^{n-1} E_{n}$.

When $E_{\max }=\infty$, since constraints in (19) and slackness conditions in (23) are satisfied, from (24), the optimal power $P_{n}^{*}$ is monotonically increasing: $P_{n+1}^{*} \geq P_{n}^{*}$. The results indicate that energy can be spread to the future for operating optimally. When the constraint in (18) is not satisfied with equality $\left(\sum_{n=1}^{l} L_{n} P_{n}<\sum_{n=0}^{l-1} E_{n}\right)$, then $\tau_{l}=0$; thus, $P_{l}^{*}=P_{l+1}^{*}$. It shows that the energy is utilized for relay nodes not only in the current slot but also in future slots. When $P_{n}^{*}<P_{n+1}^{*}$, it means that the total energy is used for current slot and no energy is used in the future.

As proven before, the harvested energy can only be used in the future, which means our algorithm allows energy to only flow to the right. To solve this problem, we propose an optimal power allocation based on directional water-filling.

It is assumed that the optimal energy at time instants $t_{1}, t_{2}, \ldots, t_{N}$ is denoted by $E_{t_{1}}, E_{t_{2}}, \ldots, E_{t_{N}}$ and is obtained before the power allocation process. The process of the algorithm is then as follows.

Step 1. Initialize the power allocation flag to 0 , and let the algorithm pointer point to the last slot $t_{N}$.

Step 2. Compare the energy level in $t_{N}$ with the energy level in $t_{N-1}$.

Step 3. No power allocation is performed when the energy level in the current slot exceeds the previous slot.

Step 4. If the energy level in the current slot is lower than the previous slot, allocate energy equally to both $t_{N}$ and $t_{N-1}$. Set the power allocation flag to 1 , mark these two slots as $t_{N-1}$, and then the algorithm pointer points to $t_{N-1}$.

Step 5. Point the algorithm pointer to the previous slot.

Step 6. If the pointer reaches $t_{1}$ and the power allocation flag is 1 , go to Step 2; if the point reaches $t_{1}$ and the flag is 0 , stop and output $P_{n}^{*}$ as the optimal power allocation; otherwise, go to Step 3.

3.2. Multirelay Selection and Session Grouping. Energy efficiency is defined as the ratio of network throughput to total power consumption. Note that (7), (8), and (17) are the functions of $G_{m}^{r_{k}}$. It is essential to consider how to put sessions into different groups and select a relay for each group. We define the session grouping results as $\Theta \in C^{K \times N \times M} . \Theta_{n}^{r_{k}, m}$ indicates whether a session belongs to $G_{m}^{r_{k}}$.

$$
\Theta_{n}^{r_{k}, m}= \begin{cases}1, & \left(s_{n}, d_{n}\right) \in G_{m}^{r_{k}} \\ 0, & \text { otherwise }\end{cases}
$$

A session $\left(s_{n}, d_{n}\right)$ can be in at most one group; thus,

$$
\sum_{k=1, m=0}^{k=K, m=M-1} \Theta_{n}^{r_{k}, m}=1 .
$$

The optimization target of session grouping is to maximize the energy efficiency in our network model. We combine (7), (8), and (17) and formulate the optimization problem as

$$
\begin{array}{ll}
\max \quad & \mathrm{EE}=\frac{\sum_{n=1}^{N} \sum_{k=1}^{K} \Theta_{n}^{r_{k}, 0} R_{D}\left(s_{n}, d_{n}\right)+\sum_{n=1}^{N} \sum_{k=1}^{K} \sum_{m=1}^{M-1} \Theta_{n}^{r_{k}, m} R_{\mathrm{NC}}\left(s_{n}, r_{k}, G_{m}^{r_{k}}\right)}{\sum_{k=1}^{K} \sum_{m=0}^{M-1} P\left(G_{m}^{r_{k}}\right)} \\
\text { s.t. } & G_{m}^{r_{k}}=\left\{\left(s_{n}, d_{n}\right), \quad \forall n, \Theta_{n}^{r_{k}, m}=1\right\} \\
& \sum_{m=0}^{M-1} \Theta_{n}^{r_{k}, m}=1, \quad\left(\forall n, s_{n} \in G_{m}^{r_{k}}\right) \\
& \Theta_{n}^{r_{k}, m} \in\{0,1\}, \quad(1 \leq n \leq N, 0 \leq m \leq M) .
\end{array}
$$


Obviously, the optimization problem in (27) is a general " $0-1$ " programming problem. However, this is a nonlinear problem that can be solved only by exhaustive search. Since each source node is expected to transmit data to its destination node from $K$ available channels, either with or without the assistance of a relay node, the complexity of exhaustive search is

$$
C_{\text {exhaustive }}=\left(2^{M} K\right)^{N}
$$

From (28), the complexity increases exponential with the number of sessions, which is intolerable when the number of sessions is large. To solve this problem, a joint relay selection and session grouping scheme is proposed based on QPSO.

QPSO is a novel multiagent optimization system modified by PSO that is inspired by the social behavior of agents. Each agent, called quantum particle, files in a $2 \mathrm{~N}$ dimensional space according to the historical experiences of itself and of colleagues. $2 \mathrm{~N}$ represents the dimension of the optimization problem $[20,21]$.

We define the position of the $j$ th quantum particle as

$$
\mathbf{L}_{j}=\left[\begin{array}{l}
\overrightarrow{\mathbf{U}} \\
\overrightarrow{\vec{V}}
\end{array}\right]=\left[\begin{array}{llll}
\mu_{j 1} & \mu_{j 2} & \cdots & \mu_{j N} \\
\varepsilon_{j 1} & \varepsilon_{j 2} & \cdots & \varepsilon_{j N}
\end{array}\right],
$$

where $\mu_{j n}$ represents the relay number of session $\left(s_{n}, d_{n}\right)$ and $\varepsilon_{\text {jn }}$ represents the group number of session $\left(s_{n}, d_{n}\right)$. In QPSO algorithm, $\mu_{j n}$ and $\varepsilon_{j n}$ are mapped into quantum particle's bit position which can be expressed as

$$
\begin{aligned}
& x_{j n}=k_{\min }+\mu_{j n}\left(k_{\max }-k_{\min }\right), \\
& y_{j n}=m_{\min }+\varepsilon_{j n}\left(m_{\max }-m_{\min }\right),
\end{aligned}
$$

where $k_{\max }$ and $k_{\min }$ are the upper bound and lower bound of the relay node number, respectively. Similarly, $m_{\max }$ and $m_{\text {min }}$ express the upper bound and lower bound of the group number.
From (30), the quantum particle's bit position is in the space of $[1, K] \times[0, M]$. Therefore, $(29)$ can be simplified as

$$
\left[\begin{array}{l}
\vec{X} \\
\vec{Y}
\end{array}\right]=\left[\begin{array}{llll}
x_{j 1} & x_{j 2} & \cdots & x_{j N} \\
y_{j 1} & y_{j 2} & \cdots & y_{j N}
\end{array}\right] \text {. }
$$

We use the integral function round $(\cdot)$ to find the optimal $\Theta_{n}^{r_{k}, m}$. Thus, the expression of $\Theta_{n}^{r_{k}, m}$ is shown as

$$
\begin{aligned}
& \Theta_{n}^{r_{k}, m} \\
& \quad= \begin{cases}1, & \text { if }\left(\operatorname{round}\left(x_{j n}\right)=k, \text { round }\left(y_{j n}\right)=m\right), \\
0, & \text { otherwise. }\end{cases}
\end{aligned}
$$

We set the objective function in (27) as the fitness function. The local optimal bit position of the $j$ th quantum particle is

$$
\left[\begin{array}{l}
\overrightarrow{\mathbf{P}} \\
\overrightarrow{\overrightarrow{\mathbf{Q}}}
\end{array}\right]=\left[\begin{array}{llllll}
p_{j 1} & p_{j 2} & p_{j 3} & p_{j 4} & \cdots & p_{j N} \\
q_{j 1} & q_{j 2} & q_{j 3} & q_{j 4} & \cdots & q_{j N}
\end{array}\right] .
$$

The global optimal bit position of the whole quantum particle population is

$$
\left[\begin{array}{l}
\overrightarrow{\mathbf{P}_{g}} \\
\overrightarrow{\overrightarrow{\mathbf{Q}_{g}}}
\end{array}\right]=\left[\begin{array}{llllll}
p_{g 1} & p_{g 2} & p_{g 3} & p_{g 4} & \cdots & p_{g N} \\
q_{g 1} & q_{g 2} & q_{g 3} & q_{g 4} & \cdots & q_{g N}
\end{array}\right] .
$$

In this proposed algorithm, we consider the generation in two dimensions. The quantum rotation angles are calculated by

$$
\begin{aligned}
& \theta_{j n}^{i+1}=e_{1}\left(p_{j n}^{i}-\mu_{j n}^{i}\right)+e_{2}\left(p_{g n}^{i}-\mu_{j n}^{i}\right), \\
& \varphi_{j n}^{i+1}=e_{4}\left(q_{j n}^{i}-\varepsilon_{j n}^{i}\right)+e_{5}\left(q_{g n}^{i}-\varepsilon_{j n}^{i}\right),
\end{aligned}
$$

where $e_{1}$ and $e_{2}$ express the relative important degree of $\overrightarrow{\mathbf{P}}$ and $\overrightarrow{\mathbf{P}_{g}}$, and the updating process of the quantum particle can be expressed as

$$
\begin{aligned}
& \mu_{j n}^{i+1}= \begin{cases}\sqrt{1-\left(\mu_{j n}^{i}\right)^{2}}, & \text { if }\left(p_{j n}^{i}=\mu_{j n}^{i}=p_{g n}^{i}, e_{3}<c_{1}\right), \\
\left|\mu_{j n}^{i} \cos \theta_{j n}^{i}-\sqrt{1-\left(\mu_{j n}^{i}\right)^{2}} \sin \theta_{j n}^{i}\right|, & \text { others, }\end{cases} \\
& \varepsilon_{j n}^{i+1}= \begin{cases}\sqrt{1-\left(\varepsilon_{j n}^{i}\right)^{2}}, & \text { if }\left(q_{j n}^{i}=\varepsilon_{j n}^{i}=q_{g n}^{i}, e_{6}<c_{2}\right), \\
\left|\varepsilon_{j n}^{i} \cos \varphi_{j n}^{i}-\sqrt{1-\left(\varepsilon_{j n}^{i}\right)^{2}} \sin \varphi_{j n}^{i}\right|, & \text { others, }\end{cases}
\end{aligned}
$$


where $e_{3}$ and $e_{6}$ are uniform random number between 0 and 1 and $c_{1}$ and $c_{2}$ are mutation probabilities between 0 and $1 / N$.

Assumption 1. The power allocation process has finished.

Assumption 2. All the channel state information (CSI) is a priori known.

Therefore, the process of QPSO algorithm is given in the following steps.

Step 1. Initialize quantum particle population, including the quantum particles' bit positions $L_{j}$ and the local optimal bit positions. Calculate the fitness of each quantum particle, and record the global optimal bit position from (27).

Step 2. Update the rotation angle for each quantum particle from (35). Update the bit positions for each quantum particle from (36).

Step 3. Calculate the fitness of all quantum particles based on updated positions.

Step 4. Update the local optimal quantum position for each quantum particle and the global optimal quantum position.

Step 5. If it reaches the predefined value of the maximum generation, stop the process, output the global optimal quantum position, and map it into the final transmission strategy by (30), (31), and (32); if not, go to Step 3.

\section{Numerical Results}

In this section, we show the simulation results to evaluate the performance of our proposed approach. As shown in Figure 1, we consider our simulation within a $1000 \times 1000 \mathrm{~m}^{2}$ square area. We consider a network randomly consisting of 50-200 sessions and 5-50 relays. For all network instances, we assume that the transmission power of each source node is $1 \mathrm{~W}$ and that the channel bandwidth is $22 \mathrm{MHz}$. The loss exponent $\alpha$ is 4 . We assume that the white Gaussian noise at all nodes has a variance of $10^{-10} \mathrm{~W}$ [8]. The circuit power for each node is chosen as $110 \mathrm{~mW}$. For QPSO, we set the maximal generation as $200, h=20, e_{1}=0.15, e_{2}=0.2, e_{4}=0.6, e_{5}=0.7$, $c_{1}=1 /(500 K)$, and $c_{2}=1 /(1000 M)$. Each result is obtained from averaging over 50 realizations. Simulation parameters are provided in Table 1.

\subsection{System Performance of Relay Node Selection and Session} Grouping. First, we consider the system performance of relay node selection and session grouping. We analyze the energy efficiency, total throughput, and power consumption versus the number of sessions. The traditional direct transmission (DT) and opportunistic relay-selected cooperative transmission (CR) are used to provide performance reference for our proposed (NC) scheme. In addition, power allocations are preoptimized in DT, CR, and NC.

In CR and NC, the source node $S_{n}$ can exploit a relay node based on the energy level at relay node and channel state
TABLE 1: Simulation parameters.

\begin{tabular}{lc}
\hline Parameters & Value \\
\hline Number of sessions & $50-200$ \\
Number of relay nodes & $5-50$ \\
Distribution area & $1000 \times 1000 \mathrm{~m}^{2}$ \\
Channel bandwidth & $22 \mathrm{MHz}$ \\
Loss exponent & 4 \\
Transmit power of source node & $1 \mathrm{~W}$ \\
Circuit power & $110 \mathrm{~mW}$ \\
White Gaussian noise & $1 \times 10^{-10} \mathrm{~W}$ \\
Number of quantum particles & 20 \\
Maximal generation & 200 \\
Rotating factor of $\theta$ & $e_{1}=0.15, e_{2}=0.2$ \\
Rotating factor of $\varphi$ & $e_{4}=0.6, e_{5}=0.7$ \\
Mutation probability & $c_{1}=1 /(500 \mathrm{~K})$ \\
& $c_{2}=1 /(1000 \mathrm{M})$ \\
\hline
\end{tabular}

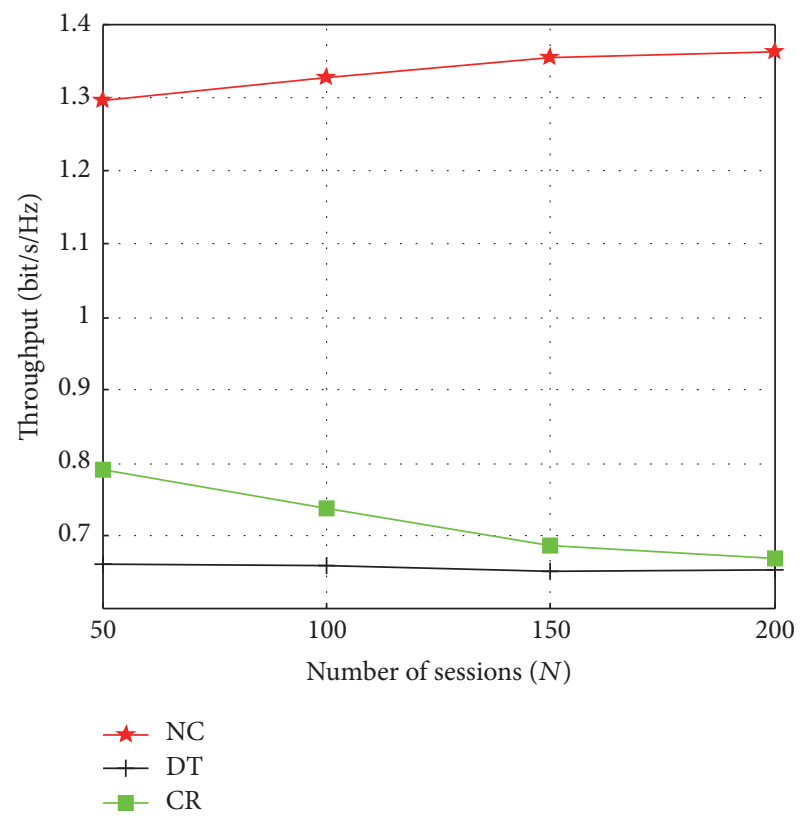

FIGURE 2: Throughput for three transmission schemes.

information (CSI) when it sends data to its destination node. Since the bandwidths of different schemes may be different, the performance is measured in $\mathrm{bit} / \mathrm{s} / \mathrm{Hz}$.

Figure 2 shows that the proposed scheme outperforms other two schemes apparently. Increasing number of sessions will increase the possibility of sessions being a reasonable group. As the number of sessions increases, the average throughput of our scheme increases as well. However, in a big number regime, the average throughput is saturated. This is because the increasing $\mathrm{NC}$ noise will affect the throughput.

Figure 3 is the average power consumption of each transmission scheme. It shows that DT consumes the most power. 


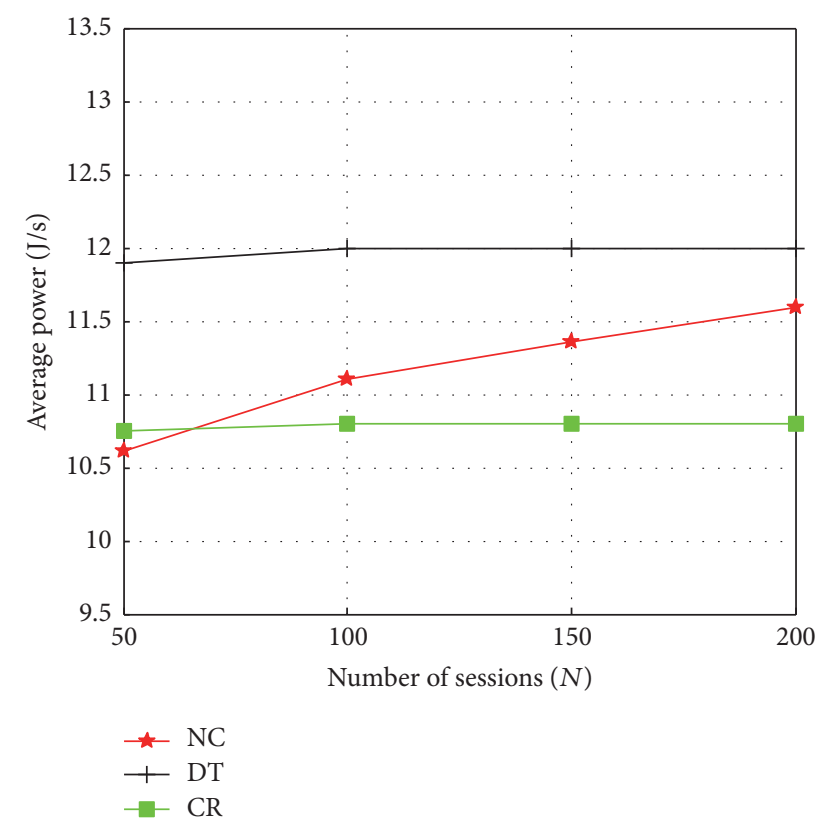

Figure 3: Average power consumption for three transmission schemes.

The reason is that this scheme is transmitting the maximum number of sessions by occupying all possible channels to improve the energy efficiency. This figure also indicates that the average power of $\mathrm{CR}$ transmission is the minimum. It is because better cooperative relay nodes are selected to maximize the energy efficiency. Unlike CR transmission, the circuit energy consumption is considered in our scheme. The figure indicates that NC performs better than DT.

Figure 4 shows the energy efficiency of different transmission schemes. It is observed that our proposed scheme achieves a higher energy efficiency than the other two schemes. This is because both the CSI and the energy level of relay nodes are considered in our scheme.

4.2. System Performance of Power Allocation. Next, we show the performance of power allocation for relay nodes. In Figure 5, the proposed scheme is validated. Our approach always outperforms the NC transmission without power allocation for relay nodes. In Figure 5(a), when the number of sessions $N$ increases, the throughput performance gap becomes larger because the efficient power allocation assures uniform distribution. Figure 5(b) presents the average power consumption versus the number of sessions. With increasing number of sessions, the power is continually increasing in both schemes. As shown in the figure, the power allocation scheme costs more power consumption than non-power allocation scheme, since higher throughput will consume more power. In Figure 5(c), the energy efficiency of power allocation scheme is superior to that of non-power allocation scheme. The energy efficiency begins to converge when the number of sessions becomes large, since the existence of NC noise could diminish the advantage of optimal power allocation.

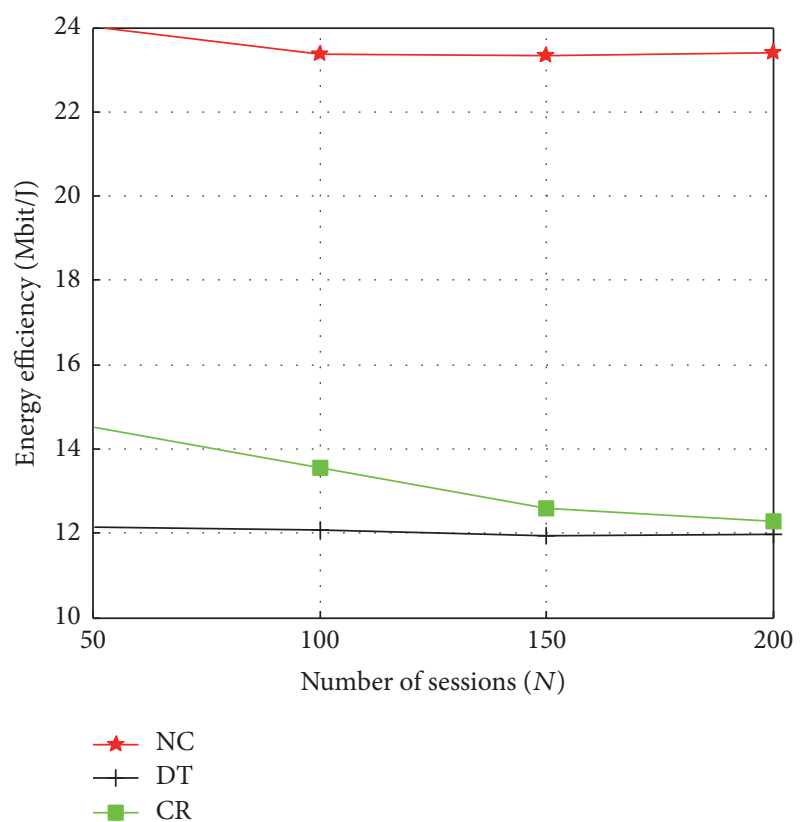

FIGURE 4: Energy efficiency for three transmission schemes.

\section{Conclusion}

In this paper, we studied the energy efficiency problem in multiple relay cooperative networks with energy harvesting. The energy efficiency is taken as the classic network throughput per power allocation. In the optimization problem, network coding noise and receiver circuit power consumption were considered. This problem can be decomposed into a power allocation problem and an energy-efficient transmission optimization problem. We solved the first problem by proposing a time-power allocation algorithm based on directional water-filling algorithm. The second problem was solved by jointly considering relay selection scheme and session grouping based on quantum particle swarm optimization. Numerical results verified that the proposed transmission scheme effectively improved energy efficiency, compared with direct transmission scheme and opportunistic cooperative transmission scheme.

\section{Competing Interests}

The authors declare that there is no conflict of interests regarding the publication of this paper.

\section{Acknowledgments}

This work was partly supported by the National Natural Science Foundation of China (61461029 and 61561032), the Natural Science Foundation of Jiangxi Province (20114ACE00200, 20161BAB202043, 20151BBE50054, and 20153BCB23020), China Postdoctoral Science Foundation Funded Project 


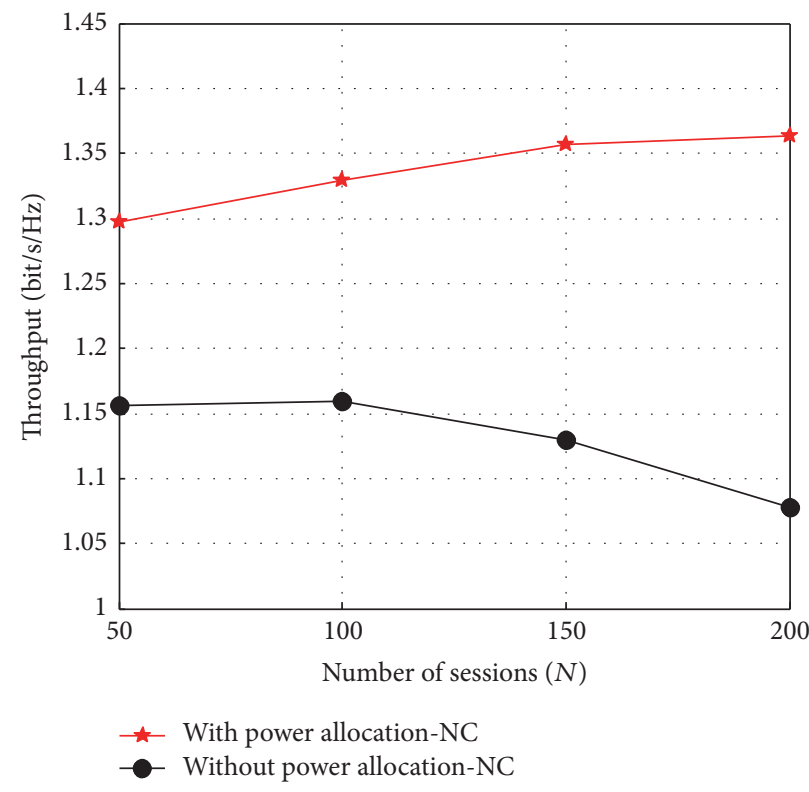

(a)

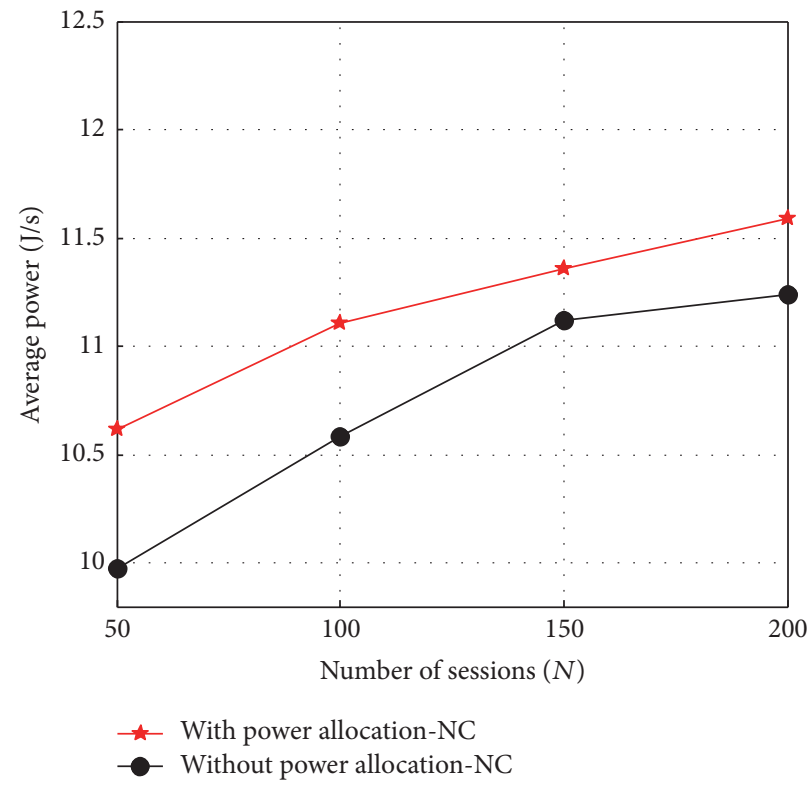

(b)

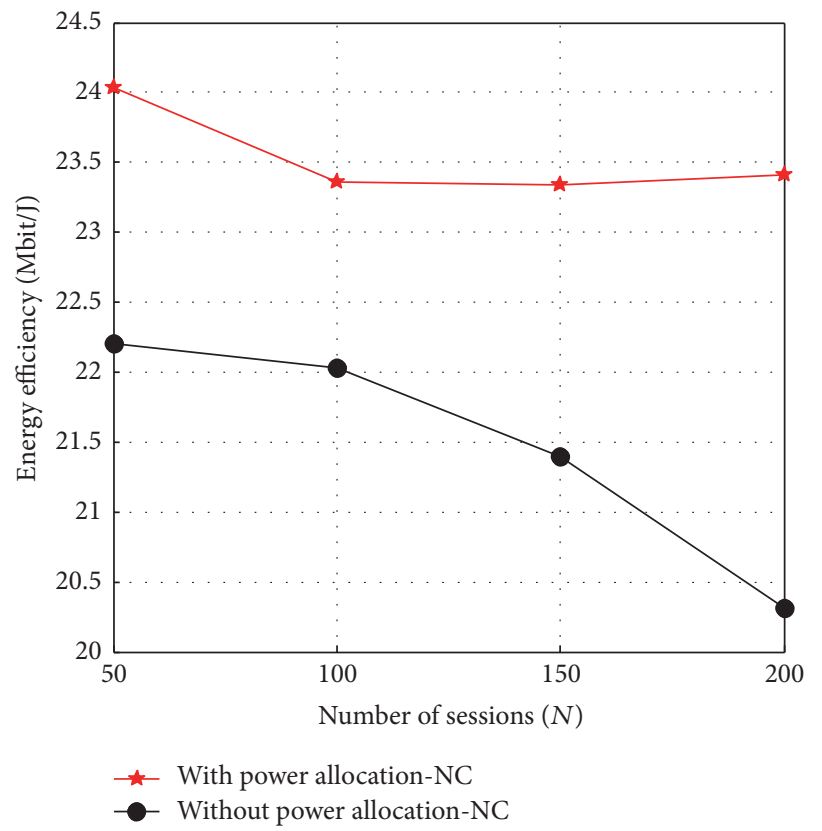

(c)

FIGURE 5: System performance of the policy with power allocation for relay nodes.

(no. 2015T80692), and Graduate Student Innovation Special Funds of Nanchang University (cx2016264).

\section{References}

[1] R. Madan, N. B. Mehta, A. F. Molisch, and J. Zhang, "Energyefficient cooperative relaying over fading channels with simple relay selection," IEEE Transactions on Wireless Communications, vol. 7, no. 8, pp. 3013-3025, 2008.

[2] A. Nosratinia, T. E. Hunter, and A. Hedayat, "Cooperative communication in wireless networks," IEEE Communications Magazine, vol. 42, no. 10, pp. 74-80, 2004.
[3] Z. Zhang, Y. Li, K. Huang, and C. Liang, "Energy efficiency analysis of energy harvesting relay-aided cooperative networks," in Proceedings of the 13th International Symposium on Modeling and Optimization in Mobile, Ad Hoc, and Wireless Networks (WiOpt '15), pp. 1-7, IEEE, Mumbai, India, May 2015.

[4] S. Sharma, Y. Shi, J. Liu, Y. T. Hou, and S. Kompella, "Is network coding always good for cooperative communications?" in Proceedings of the IEEE INFOCOM, pp. 1-9, San Diego, CA, USA, March 2010.

[5] S. Sharma, Y. Shi, J. Liu, Y. T. Hou, S. Kompella, and S. F. Midkiff, "Network coding in cooperative communications: friend or foe?" IEEE Transactions on Mobile Computing, vol. 11, no. 7, pp. 1073-1085, 2012. 
[6] Z. Mobini, P. Sadeghi, and S. Zokaei, "Network coding noise reduction via relay power allocation in a two-unicast wireless system," in Proceedings of the IEEE 22nd International Symposium on Personal, Indoor and Mobile Radio Communications (PIMRC '11), pp. 1459-1464, IEEE, Toronto, Canada, September 2011.

[7] Z. Mobini, P. Sadeghi, M. Khabbazian, and S. Zokaei, "Power allocation and group assignment for reducing network coding noise in multi-unicast wireless systems," IEEE Transactions on Vehicular Technology, vol. 61, no. 8, pp. 3615-3629, 2012.

[8] S. Sharma, Y. Shi, Y. T. Hou, H. D. Sherali, and S. Kompella, "Joint optimization of session grouping and relay node selection for network-coded cooperative communications," IEEE Transactions on Mobile Computing, vol. 13, no. 9, pp. 2028-2041, 2014.

[9] M. Zhou, Q. Cui, R. Jäntti, and X. Tao, "Energy-efficient relay selection and power allocation for two-way relay channel with analog network coding," IEEE Communications Letters, vol. 16, no. 6, pp. 816-819, 2012.

[10] K. Xiao, Y. Zhang, G. Feng, X. Duan, J. Zhu, and Z. Liu, "eCOPE: energy efficient network coding scheme in multi-rate wireless networks," in Proceedings of the IEEE Wireless Communications and Networking Conference Workshops (WCNCW '13), pp. 1823, Shanghai, China, April 2013.

[11] G. Zhang and Y. Li, "Cooperative multicast transmission strategy for energy-efficient dynamic network coding," in Proceedings of the IEEE International Conference on Communications Workshops (ICC '13), pp. 479-483, IEEE, Budapest, Hungary, June 2013.

[12] H. Wang, W. Wang, and Z. Zhang, "Opportunistic wireless information and energy transfer for sustainable cooperative relaying," in Proceedings of the IEEE International Conference on Communications (ICC '15), pp. 160-165, London, UK, June 2015.

[13] K. Tutuncuoglu and A. Yener, "Cooperative energy harvesting communications with relaying and energy sharing," in Proceedings of the IEEE Information Theory Workshop (ITW '13), IEEE, Angeles, Calif, USA, September 2013.

[14] K. H. Liu, "Performance analysis of relay selection for cooperative relays based on wireless power transfer with finite energy storage," IEEE Transactions on Vehicular Technology, vol. 65, no. 7, pp. 5110-5121, 2016.

[15] I. Ahmed, A. Ikhlef, R. Schober, and R. K. Mallik, "Joint power allocation and relay selection in energy harvesting AF relay systems," IEEE Wireless Communications Letters, vol. 2, no. 2, pp. 239-242, 2013.

[16] N. Zhao, R. Chai, Q. Hu, and J. Zhang, "Energy efficiency optimization based joint relay selection and resource allocation for SWIPT relay networks," in Proceedings of the 10th International Conference on Communications and Networking in China (ChinaCom '15), pp. 503-508, Shanghai, China, August 2015.

[17] N. Zhao, R. Chai, Q. Hu, and J.-K. Zhang, "Energy efficiency optimization based joint relay selection and resource allocation for SWIPT relay networks," in Proceedings of the 10th International Conference on Communications and Networking in China (ChinaCom '15), pp. 503-508, Shanghai, China, August 2015.

[18] X. Shen, T. Zhang, Z. Zeng, and X. Lin, "Energy efficient session grouping scheme in network-coded cooperative communications," in Proceedings of the 10th International Conference on Communications and Networking in China (ChinaCom '15), pp. 158-162, Shanghai, China, August 2015.

[19] O. Ozel, K. Tutuncuoglu, J. Yang, S. Ulukus, and A. Yener, "Transmission with energy harvesting nodes in fading wireless channels: optimal policies," IEEE Journal on Selected Areas in Communications, vol. 29, no. 8, pp. 1732-1743, 2011.

[20] J. Cao, T. Zhang, Z. Zeng, Y. Chen, and K. K. Chai, "Multi-relay selection schemes based on evolutionary algorithm in cooperative relay networks," International Journal of Communication Systems, vol. 27, no. 4, pp. 571-591, 2014.

[21] T. Zhang, J. Cao, Z. Zeng, and D. Liu, "Joint relay selection and spectrum allocation scheme in cooperative relay networks," in Proceedings of the 79th IEEE Vehicular Technology Conference (VTC '14), pp. 1-6, IEEE, Seoul, Republic of Korea, May 2014. 

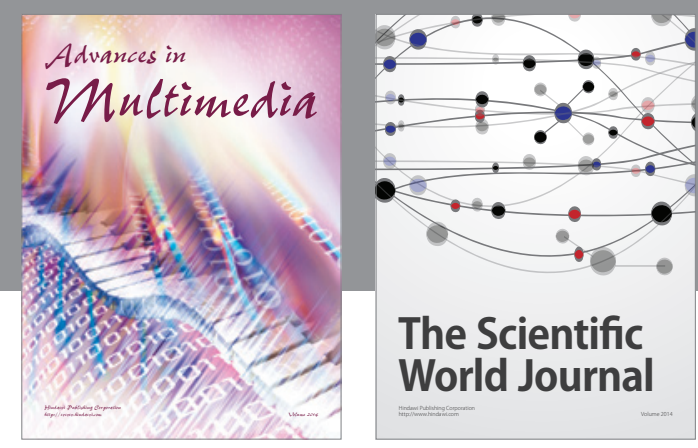

The Scientific World Journal
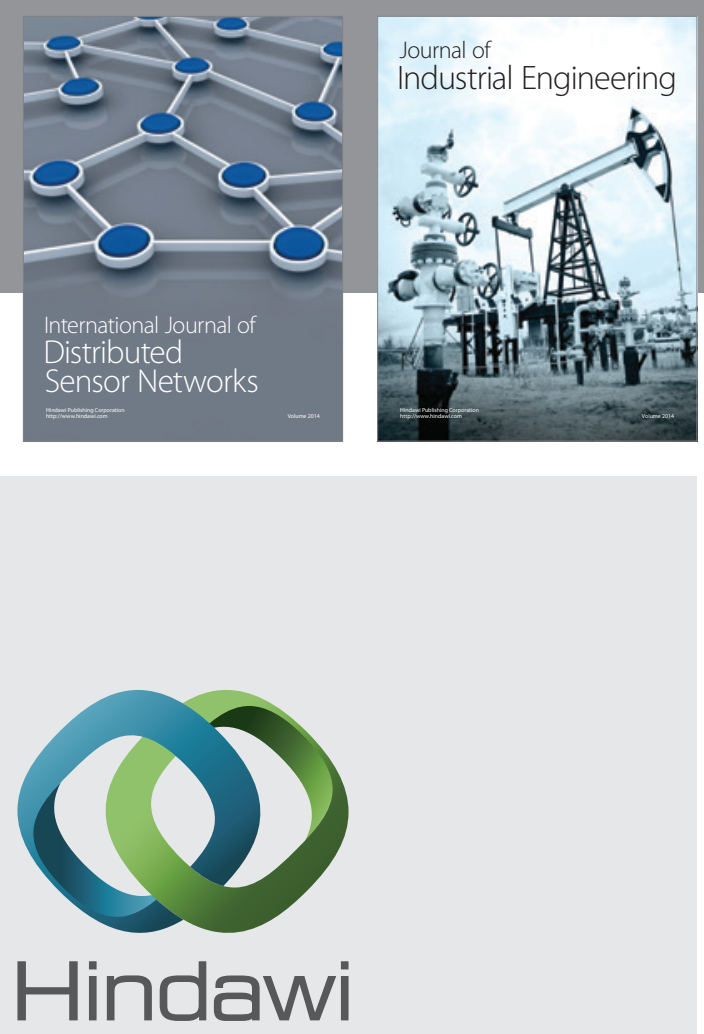

Submit your manuscripts at

http://www.hindawi.com

\section{Computer Networks} and Communications
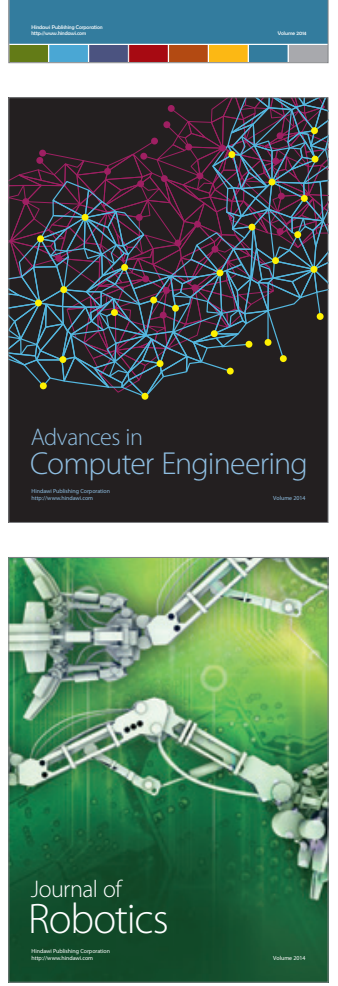
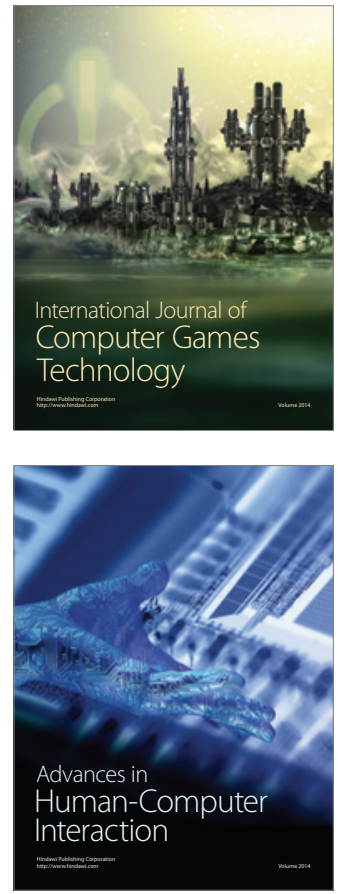
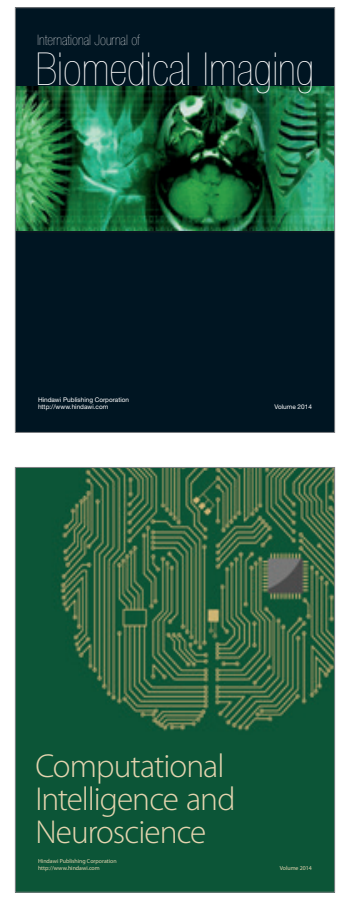
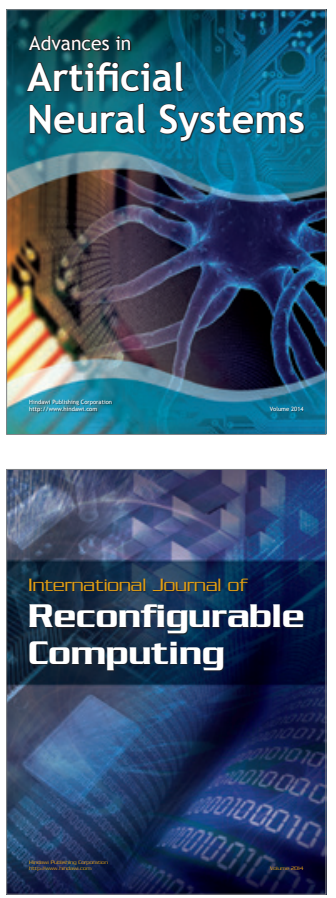
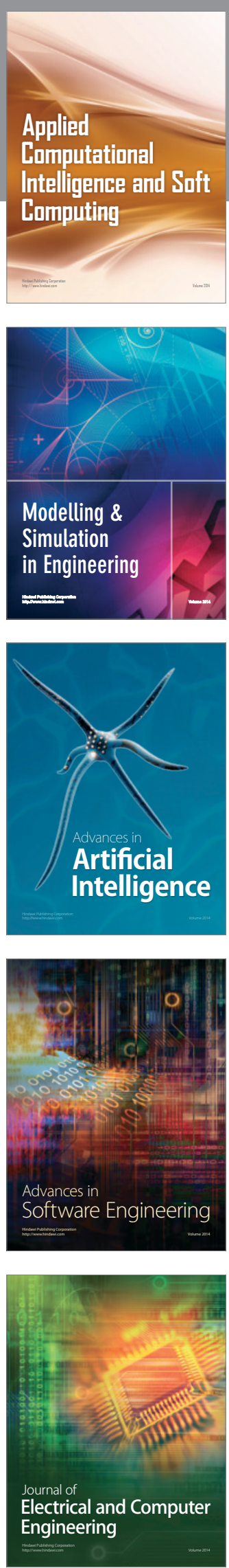